\title{
Thickened tailings deposition for closure
}

\author{
Nicholas Thompson SRK Consulting (Australasia) Pty Ltd, Australia \\ Pepe Moreno SRK Consulting (Australasia) Pty Ltd, Australia
}

\section{Abstract}

The strategic implementation of a thickened tailings system for an existing conventional TSF can be beneficial in terms of significantly reducing the required post-operational earthworks and the time to achieve final closure.

One of the most challenging aspects of the successful closure of a TSF is the long-term water management of the tailings surface. A typical concave-shaped, conventional paddock TSF could be transitioned to have a convex, thickened tailings surface towards the end of operations through modifying the tailings disposal strategy to create a water-shedding final landform, limiting the amount of surface re-shaping and borrow material required. This would also maximise the tailings storage capacity while generating a facility profile that would be significantly more amenable to closure.

Challenges that would need to be explored and assessed before implementing such a strategy relate to the change from conventional to thickened tailings deposition and include characterising the slurry, adjusting the disposal methodology to include central discharge, assessing the impact of depositing thicker tailings over the existing beach, and modifying the supernatant water management plan.

This paper discusses the concept of 'deposition for closure' using thickened tailings that is currently relatively uncommon worldwide; however, is becoming increasingly attractive due to more stringent closure requirements and enhanced thickening technology. The implementation of this concept is currently in the early stages for a TSF at a gold mine in Ghana, with the knowledge gained from this project used as the basis for presenting the potential advantages of such a strategy, as well as the likely issues and challenges encountered.

\section{Introduction}

With the mining industry transitioning through a well-publicized downturn as a result of lower commodity prices and unstable macro-economic conditions around the world, mine owners have sharpened their focus on implementing innovative concepts while still minimizing unnecessary expenditure. The management of tailings, already perceived by many as simply a cost burden to the mine, is one of the key areas that can be targeted for savings.

Water management of the tailings storage facility (TSF) is one of the most important drivers when designing tailings disposal strategies, and post-operational water management of the tailings surface is also one of the most challenging aspects in terms of successful closure of a TSF. However, site operators typically select tailings disposal methods based on short-term convenience, with minimal consideration given to future closure objectives or cost implications.

TSFs are normally the largest disturbed area at a mine site and therefore incur the highest cost to rehabilitate. Profits made during the life of a mine can be gradually eroded if closure is delayed or problematic due to a 
strategic closure plan not being progressively implemented during operations.

The transition from operations to closure of a TSF brings about numerous challenges that need to be dealt with to achieve specific closure objectives and create a final stable facility. For example, it is not uncommon to see conventional paddock-style TSFs that cannot be closed immediately after the end of operations due to the tailings surface being very soft and unconsolidated with a large centrally ponded area that has not achieved sufficient strength. These facilities often require a significant amount of borrowed fill material to attain a final shape that is compatible with the site closure objectives.

Appropriately dealing with these challenges often translates into low confidence when applying conventional closure strategies, such as covers and capping layers, to support early conceptual closure plans for ongoing financial assurance assessment. Additionally, large capital expenditure and delays during the closure process at the end of the operating life of the facility can be encountered.

Owners and designers of existing conventional TSFs can utilize the many benefits of dewatering techniques to assess the feasibility of integrating thickened tailings into their current conventional disposal system with closure in mind.

Potential closure advantages are a key opportunity in addition to the more well-known improved water recovery and enhanced storage capacity.

Post-operational earthworks requirements and time to achieve closure can be significantly reduced by modifying the tailings disposal strategy towards the end of operations by utilizing thickened tailings to create a convex-shaped, water-shedding final landform (via central discharge), limiting the amount of postoperational surface re-shaping and cover borrow material required. This may considerably reduce the time for implementation of closure of the facility and overall site rehabilitation, as the overlying tailings would consolidate faster once the convex shape is attained.

There are challenges that need to be examined and assessed before embarking on a closure strategy that makes use of thickened tailings deposition - these mostly relate to retrofitting the plant and TSF from a conventional tailings to a thickened tailings operation. These challenges include characterising the slurry, adjusting the disposal methodology to include central discharge, assessing the impact of depositing thicker tailings over the existing beach, and modifying the supernatant water management plan.

The concept of 'deposition for closure' using thickened tailings is explored, with a case currently in the early stages of implementation for a TSF at a gold mine in Ghana used as the basis for initiating the concept. This case considers a range of technical, environmental, operational, closure and regulatory aspects. With the knowledge gained from this assessment as well as a review of existing operations, this paper presents the potential advantages that such a strategy may bring to an operation and discusses some of the key issues and challenges to consider prior to implementation.

\section{Deposition for closure strategy overview}

Full perimeter discharge is a common disposal method for conventional TSFs. It generally creates a concaveshaped surface leading to a centrally located supernatant pond remaining at the end of operations. A typical schematic layout of this configuration is shown in Figure 1. 


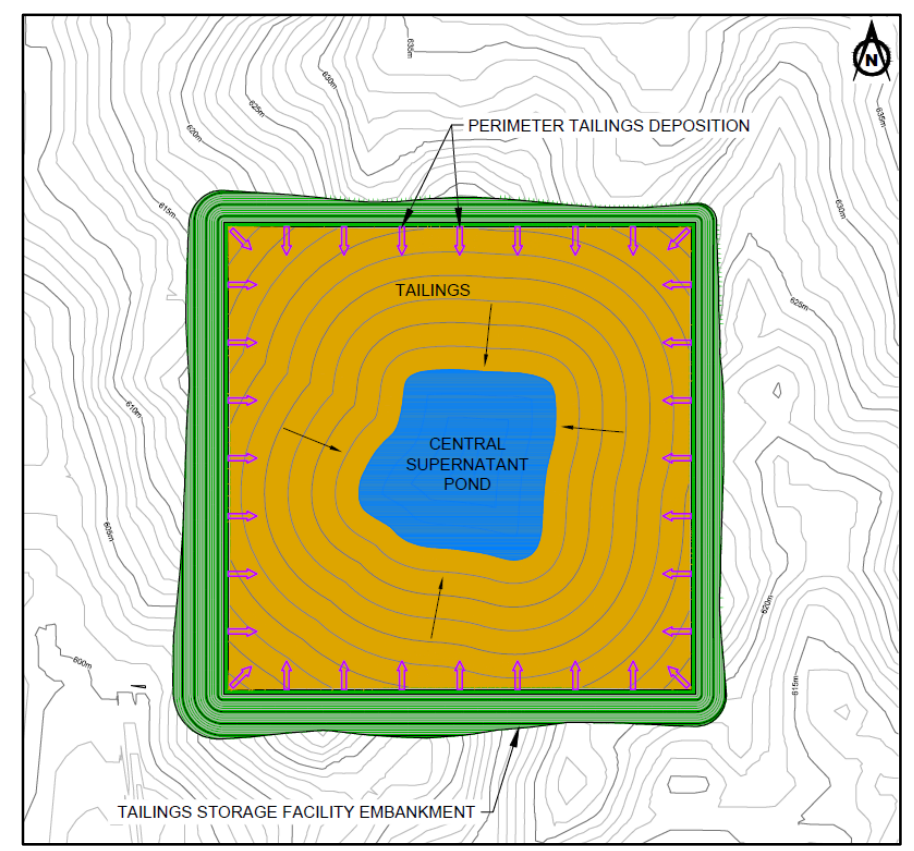

Figure 1 Schematic TSF with conventional perimeter tailings deposition and central supernatant pond

The transition from operations to closure requires changing the water containment requirements for the facility, with closure objectives generally focused on limiting the amount of water remaining on the tailings surface post operations. A common technique is to construct a cover above the final tailings surface, which is shaped to shed clean runoff water away from the facility towards a controlled spilling zone, rather than pool on the upper surface. To construct the cover and change this concave surface to a final convex landform to promote water shedding, a significant amount of fill material (waste rock or borrow) is required. The steeper the beach slope, the greater the required volume of material.

The alternative strategic approach that is the subject of this paper involves re-shaping the TSF during operations using tailings to create the final landform with the inclusion of central discharge. This is done by modifying the deposition strategy so that thickened tailings are disposed in targeted areas in the latter stages of the mine life, as opposed to uncontrolled deposition for the simple purpose of filling the basin.

The strategic deposition of tailings (from both central and perimeter locations) can lead to considerable cost savings in the long term if the site-specific conditions are conducive to such a transition. Implementation of this concept can significantly reduce post-operational earthworks and construction requirements, and decrease the time to achieve final rehabilitation of the site; the timing aspect is often critical.

The selected deposition strategy would be strongly influenced by the exact shape of the facility. However, typically the tailings would be deposited radially around the TSF from a slightly eccentric location, creating a beach grading towards the dedicated spilling outlet. Depending on the shape and configuration of the TSF, it may also be necessary to discharge tailings from strategic locations around the perimeter of the facility, so that a consistent beach slope to effectively convey the water towards a low point acting as a controlled spilling zone is achieved.

A schematic layout of this revised deposition strategy which makes use of central and perimeter tailings discharge is shown in Figure 2. 


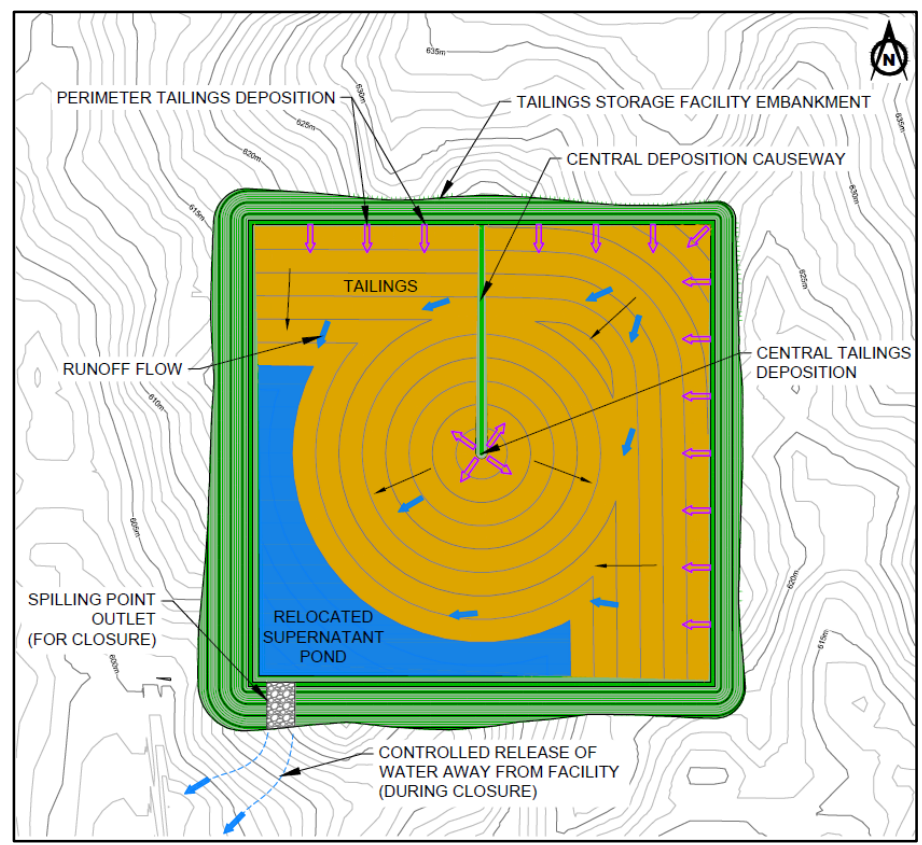

Figure 2 Schematic TSF with revised deposition strategy by means of which central and perimeter tailings discharge creates a water-shedding surface directing runoff towards a controlled spilling outlet

In most cases, a cover layer, typically composed of fill material and growth media, would still be required for isolation and environmental purposes; however, because the underlying tailings surface is already formed to the desired shape, the cover material required would essentially be a thin layer of fill, as opposed to extensive bulk filling.

The controlled water-shedding surface would also limit potential overtopping issues and minimize ongoing infiltration of rainfall from a pond into the tailings mass, promoting faster drawdown of the phreatic level inside the tailings mass and limiting future seepage concerns.

The use of a water-shedding surface significantly reduces the required lag period between the end of final deposition and the start of cover placement. The central area (where the pond is usually located) is normally much less consolidated than the rest of the TSF and therefore more susceptible to a large degree of settlement. As the pond is displaced away from the central position, consolidation of this area is promoted by self-weight, and it can effectively reach the same level of consolidation as the perimeter zones in a much shorter timeframe than would otherwise occur. Thus, in most cases, trafficability would be improved and the closure cover over the tailings surface could be placed in a shorter timeframe after the end of operations, as the tailings beach would be dryer and would have a higher degree of consolidation and strength.

\section{$3 \quad$ Existing operations adopting similar concept}

While this specific 'deposition for closure' strategy is not currently common practise across the industry and limited public information being available, there are some known cases across the world that have already, or are in the process of, modifying their tailings disposal strategy at existing conventional facilities. While there are varying methods and approaches across the different operations, the general concept of strategically depositing thickened tailings, with closure in mind, is consistent.

The Tanami and Union Reefs gold operations in Australia both incorporated thickened tailings disposal towards the end of operations with the aim of reshaping the TSF landforms to minimize rehabilitation earthworks required for closure. As is the intent with this paper's case study operation, the Tanami operation implemented central thickened discharge in the basin of the paddock facility to change the overall tailings 
surface slope from a concave to a convex profile to maximize capacity and form a shedding surface that allows fast-tracking of closure objectives. The Union Reefs operation's intent was primarily to change the basin profile from upstream sloping to downstream sloping, thereby maximising capacity. The top surface of both facilities has been reformed via strategic tailings deposition in such a way that runoff is directed to a controlled spilling outlet that is amenable to long-term management (Robins, 2004).

The Kimberley Diamond Mines operation in South Africa used the deposition of thickened tailings for rehabilitation of old slimes dams in the latter stages of mining to maximize tailings storage capacity while generating a final landform profile that would be more suitable for closure. Due to the low rainfall, a key focus of this operation was to minimize water usage; thus, the benefit of thickened tailings in terms of lower water consumption, was also realized. The overall benefits of implementing this change were considered to outweigh the costs related to the dewatering system, including installation of the new thickener and positive displacement pumps at the treatment plant (Robins, 2004).

More recently, the Prestea Gold Mine in Ghana implemented a central discharge strategy onto an existing conventional tailings paddock dam with the intention of maximizing tailings storage in the lead up to closure. The tailings were thickened and deposited from an elevated platform in the center of the facility, using waste rock to construct the access spine across the facility. This strategy created challenges with pond water management as surface water was not directed to a controlled spilling outlet, with the existing perimeter embankments not designed or constructed to retain ponded water.

The Peña Colorada iron ore mine in Mexico is currently in the process of commissioning thickened tailings discharge to replace the current conventional deposition, with a focus on maximizing the existing disturbed footprint, establishing a steeper beach slope (to enhance capacity) and reducing the volume of water pumped back to the plant. Challenges with this transition have included the high seismicity of the area and the high rate of rise at which the underlying conventional tailings were placed. This will likely result in significant settlement of the tailings and could also potentially lead to the thickened tailings beach slumping into the conventionally deposited tailings after a seismic event (Moreno et al., 2014).

In general, the existing cases that have adopted the concept of depositing thickened tailings into an existing conventional tailings facility have implemented the change due to the many perceived potential benefits it provides, with a focus on closure cost savings in addition to simply operational improvements. Each operation has considered that it is financially viable over the long term to compensate for the initial capital investment; a viewpoint which would likely initially draw skepticism given operations are in their latter stages.

However, there are potential risks related to implementing the thickened tailings deposition for closure strategy and due consideration of these risks is critical; it is important to understand site-specific conditions and the operational history of the TSF to design a landform which successfully evolves over time towards closure.

\section{$4 \quad$ Key considerations of modifying disposal strategy}

\subsection{Tailings composition and characterisation}

Characterisation of the slurry should be conducted as early as possible before making the significant financial investment involved in changing a tailings disposal system.

It is critical that the chemical composition of the thickened tailings product is well understood prior to implementing a direct water-shedding strategy. Adopting a water-shedding tailings upper surface is only feasible if the upper exposed portion of the tailings is chemically benign and stable, and will not have an impact on the receiving environment. 
Typically, the intent would still be to place a cover layer above the tailings surface for long term closure. However, there will likely be an intermediate period during cover placement during which tailings would be exposed to potential oxidation, and as runoff comes in direct contact with the tailings before exiting the facility, there is a potential for contaminating the natural environment and watercourses.

The tailings would typically need to be non-acid forming and have very low potential for leaching heavy metals, below the applicable regulatory standards, to promote clean excess water runoff. In cases where multiple streams of tailings are being produced, it would be possible to select the most geochemically benign tailings stream and spread it exclusively as a covering layer during the latter stages of operation.

\subsection{Geotechnical stability and conventional/thickened tailings interface}

When perimeter discharge is used as the disposal strategy with the intent of creating a central supernatant pond, the containment embankments are not necessarily required to be water retaining. If a modified disposal strategy is adopted to produce a convex water-shedding tailings surface, water may temporarily pond against the existing outer embankments. The stability of these embankments needs to be assessed, with the likelihood of a higher phreatic surface forming - either through direct adjacent ponding or saturated tailings. Depending on the shear strength of the fill material and the existing downstream slope angle, mitigation measures, such as a buttress constructed at the downstream toe or elevated drains, may be required.

Depositing fresh thickened tailings over a conventional tailings deposit leads to a raft of considerations relating to the interaction between the two tailings layers, and these must be identified and evaluated. It is crucial to assess this interaction to determine the geotechnical stability (static and dynamic) of the composite landform. A secondary effect to consider is the deformation of the beach due to differential settlement, which could influence the final shape of the tailings surface in the long term.

An important consideration is assessing the current consolidation state of the existing tailings deposit, which is largely driven by its previous deposition strategy and operation. The stability of the forming beach is critical, as potential slumping of the thickened tailings into the softer (conventional) tailings may trigger a surge in pond levels; this is particularly concerning in areas of high seismicity. Limiting the tailings rate of rise could be imposed to mitigate this risk.

A comprehensive CPTu program would be required to obtain representative geotechnical profiles of the existing conventional deposit and, from these, evaluate whether there are potential stability and/or liquefaction concerns. In a general sense, tailings with a state denser than the critical state $(\psi<0)$ will be dilative and will strain harden in undrained shear, whereas tailings with a state looser than the critical state $(\psi>0)$ will be contractive and will strain soften in undrained shear. A Soil Behavior Type (SBT) chart from CPTu defining a region with critical state greater than zero could be used as a screening tool to determine areas with potential stability issues (Robertson, 2010) and from there design disposal strategies that enhance consolidation of the beach.

\subsection{Disposal strategy}

The change of disposal strategy to a central thickened discharge operation introduces additional complexity to the operations - cycling between the central and perimeter deposition to create the desired beach shape must be managed. It is important to focus on integrating and using current practices, operator capabilities and available infrastructure (such as operational decant systems, pipelines etc.) where possible. To do so will limit the capital expenditure (excluding dewatering equipment) in terms of purchasing new and/or relocating existing infrastructure, and increase the confidence of the TSF operators in managing the revised deposition strategy, making the transition as smooth as possible. 
A review of the local drainage network around the TSF is required to determine the optimal location for the spilling outlet and water release, and to allow linkage to a network of streams (if possible), avoiding any downstream infrastructure or local villages near the mine. If the rest of the mine is continuing operation beyond this TSF, the location of existing mine infrastructure, including ponds, roads, pipes and control instrumentations, would need to be considered when locating the spilling point for release of runoff after closure.

\subsection{Existing closure plan}

Depending on the extent to which the deposition strategy and water management are intended to be changed, there may be some instances where a modification of the approved closure plan is required. As closure plans are commonly approved prior to, or in the early stages of, operations, changing to a thickened tailings deposition method may provide an opportunity to introduce a much more efficient reclamation plan, which is updated based on the current improved status and condition of the TSF, which, in turn, may enable earlier relinquishment.

\section{$5 \quad$ Implementation of tailings deposition for closure strategy}

Adopting a thickened tailings strategy, switching from conventional deposition, requires a staged approach which carefully considers key criteria as discussed above.

The following sections discuss a logical sequence to safely implement the proposed closure strategy. Each stage aims to progressively reduce the uncertainty of the inputs for the following stages, with costs progressively spent on investigation - advancing from simple characterisation work through to actual implementation of the strategy. This process should be considered indicative only as it is obviously very dependent on site specific features and would need to be adopted to suit.

\subsection{Characterisation of the slurry}

Characterisation of the slurry would need to include, at an early stage, geochemical, rheological and geotechnical assessment.

As discussed previously, geochemical characterisation of the slurry is fundamental to assess whether the tailings cover will be chemically stable on exposure to wet/dry cycles, as the primary aim of this strategy is to direct clean runoff away from the facility. Typical characterisation would include acid-forming potential, salt-wicking potential and leachability of heavy metals.

Rheological characterisation of the slurry provides a sound basis to establish a credible range of beach slopes that can be achieved at a certain thickening rate. This is important as it influences the shape of the final landform and informs the disposal strategy for gradually shifting from a concave to a convex surface. Tailings characterisation should include a rheogram for selected thickening rates and a yield stress versus solids concentration assessment to assist in developing beach slope modelling. At this point, the feasibility of applying in-line flocculation should also be explored if considered appropriate.

The geotechnical properties of the tailings should be assessed early in the process to evaluate suitability depending on the specific closure objectives. Important parameters include permeability (saturated and unsaturated), consolidation properties and shear strength. This would provide a first estimation of the likely behaviour of the thickened and conventional tailings interaction.

Full characterisation of the slurry and determining feasibility of this closure strategy will provide confidence in further investing in more detailed investigation, such as determining the geotechnical profiles of the basin, confirming the location of key structures, such as ponds and spillways, and routing of the spillway discharge flow. 


\subsection{Design of the thickening system}

Characterisation of the slurry would enable the design of a suitable thickening system to be undertaken integrating the design of the thickener, transport system and disposal strategy. The key link between these three disciplines is the rheology of the tailings, and the objective would be to obtain a suitable final landform shape. The selection of a beach angle that can form a sustainable convex shape would influence the thickening target for the thickener, and this, in turn, will define the inputs for design of appropriate pumps, pipeline and tailings discharge elements.

\subsection{Deposition trials to verify beach slope target}

The success of implementing this closure strategy strongly depends on achieving certain beach angles and final overall landform profiles. As this strategy is focused on implementation at an operating mine, it is therefore possible to undertake a series of deposition trials designed to observe the variability of the beach formed and, from there, define appropriate disposal strategies that may assist the tailings operator in implementing the strategy at full scale. The assumptions made during previous stages could be validated in this stage.

\subsection{Geotechnical investigation and access causeway construction}

The central thickened discharge method requires the tailings deposition pipeline to be extended to the centre of the facility, and therefore requires the construction of a purpose-built central deposition causeway above the existing tailings for operational access.

At this point, a geotechnical investigation (including CPTu) of the current tailings mass should be available to enable a suitable design of the causeway to be undertaken, as well as a stability assessment of the thickened/conventional tailings interaction.

With a conventional TSF using perimeter deposition, there will be a central supernatant pond, and therefore the tailings nearer the centre of the facility are likely to be of relatively low shear strength. This could lead to slumping of the dumped material (often tailings or waste rock) when constructing the causeway. However, experience in this type of construction is well developed - construction of the causeway could be undertaken in a similar manner to the construction of typical supernatant pool walls. Prior to construction of the causeway, the perimeter discharge would need to be adjusted to temporarily move the pond away from the proposed causeway alignment, allowing this beach area to dry out and consolidate to a workable degree.

\subsection{Commissioning central thickened discharge}

The trial deposition stage would have enabled guidelines on achieving the required landform shape to be developed; these guidelines may be refined throughout the life of the operation. It is important to note that the deposition strategy should include a detailed plan for safely migrating the operating pond towards the final spilling points, and at this initial stage, achieving a target design beach angle is a secondary focus. Once the pond has reached its targeted position, the aim of the operation should be to consistently achieve the target beach profile with closure in mind.

During the early stages of the thickened tailings deposition, the potential low shear strength of the central tailings area may lead to deformation of the existing tailings surface because of consolidation and differential settlement. Regular surveying of the tailings beach should be done to monitor progression of the tailings beach development (in comparison to the design surface) and identify any low zones requiring extra filling to promote a reasonably consistent tailings beach grading towards the identified spilling outlet. These low points can be filled by extending deposition pipes from existing spigots into the middle of the low-lying areas, filling up the small depressions in the beach with tailings as necessary. The survey would indicate the achieved beach slope and based on this operational practice and target elevations could be adjusted if necessary. 


\subsection{Water management}

Deposition of the tailings should be controlled to maintain the supernatant pond around the future spilling point, creating a low point in that area. Once tailings deposition has ceased and the design landform is attained, the pond should be temporarily shifted away from the outlet point to promote desiccation of this area, as that will allow for light equipment access to install the cover (if required) and spillway elements. A sedimentation pond may be required in the post-operational stage to prevent tailings being washed through the outlet point and to capture sediment prior to release of the water into a natural surface water stream as vegetation is being established or the TSF surface is being otherwise stabilized.

The breaching of the embankment, via spillway construction, will shift the TSF from being a water-retaining structure to a water-shedding facility, and as such, the freeboard would then constitute the distance between the maximum design water elevation (related to a design storm) in the spillway and the embankment crest elevation. Runoff water will then be evacuated via the spillway and ponding would be limited to isolated, transient pooling as water discharges over the spillway.

\section{Conclusions}

A sustainable tailings management system requires a comprehensive assessment of the full life-cycle costs of tailings disposal - costs associated with closure and long-term rehabilitation should be strongly considered, as well as the daily operational costs. Attempts at closing conventional TSFs operated with a central pond may lead to prolonged implementation periods and substantial earthworks to acquire the final landform shapes aligned with the agreed closure objectives.

Implementing a modified 'deposition for closure' approach using thickened tailings at an existing TSF towards the end of operations to create a water-shedding final surface would allow controlled management of excess water both during operations and at closure. The transformation from a concave to a convex surface using tailings would considerably reduce the borrow fill material quantities required during closure construction works by limiting the amount of surface re-shaping required to create a profile amenable to closure.

Furthermore, it reduces the required lag period between the end of deposition and the start of closure cover placement works. These factors directly translate to reductions in both cost and time expended in implementing the facility's closure.

These benefits need to be weighed against the capital cost associated with dewatering and pumping high density materials.

While there are numerous potential advantages in applying a 'deposition for closure' strategy to an existing project, it is critical to assess the various site-specific issues and challenges involved with modifying the existing tailings disposal method in a staged approach prior to implementation of the new strategy.

\section{References}

Moreno, J., Thompson, N. and Hore, C. 2014, 'Selection criteria for two alternative thickened slurries to be deposited over conventional tailings: storage capacity and liquefaction', in R.J. Jewell, A.B. Fourie, P.S. Wells and D. Van Zyl (eds), Proceedings of the 17th International Seminar on Paste and Thickened Tailings, Australian Centre for Geomechanics, Perth.

Robertson, P.K. 2010, 'Evaluation of flow liquefaction and liquefied strength using the cone penetration test', Journal of Geotechnical and Geoenvironmental Engineering, vol. 136, no. 6, pp. 842-853.

Robins, M. 2004, 'Closure of tailings facilities: current practice review and guidelines for success', Faculty of Engineering and the Built Environment. 